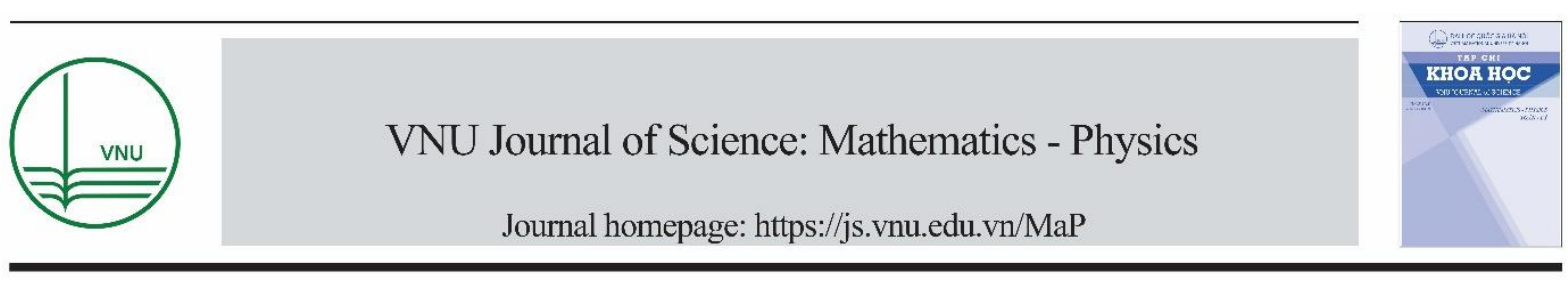

Original Article

\title{
Adsorption of 2-Butanone on Pristine Graphene: A First-principles Study
}

\author{
Phung Thi Viet Bac ${ }^{1}$, Pham Trong Lam ${ }^{1}$, Dinh Van $\mathrm{An}^{1,2,3, *}$ \\ ${ }^{I}$ Nanotechnology Program, VNU Vietnam Japan University, Vietnam National University, Hanoi, \\ Lии Нии Phиос, My Dinh, Nam Tu Liem, Hanoi, Vietnam \\ ${ }^{2}$ Institute of Science and Technology Development, Thu Dau Mot University, Binh Duong, Vietnam \\ ${ }^{3}$ Center for Atomic and Molecular Technologies, Graduate School of Engineering, \\ Osaka University, Suita, Osaka, Japan
}

Received 29 January 2020

Revised 12 February 2020; Accepted 12 February 2020

\begin{abstract}
This study investigates the adsorption mechanism of 2-butanone (ethyl methyl ketone) on the surface of graphene by using Density Functionals Theory (DFT). A 2-butanone molecule was chosen as a selected example of main volatile organic compounds (VOCs) in exhaled breath. To describe the absorption of 2-butanone and graphene substrate, the study performed DFT simulations including van de Waals (vdW) interactions implemented in the Vienna Ab-initio Simulation Package (VASP). The global minimum energy configurations and binding energies for a 2-butanone molecule adsorbed on graphene were determined by using Computational DFT-based Nanoscope tool for imaging the binding possibility of the adsorbed molecules on the graphene surface. The adsorption energy profiles were calculated by three functionals of van der Waals interactions: revPBE-vdW, optPBE-vdW, and vdW-DF2. The study results show that the adsorption energy was highly sensitive to the vdW functionals. The study also provides a detailed discussion of fundamental insights of the interactions between 2butanone and graphene through molecular doping, i.e., charge transfer.
\end{abstract}

Keywords: VOCs adsorption, 2-butanone, graphene, ab-initio calculations, charge transfer.

\section{Introduction}

Detection and monitoring of the volatile organic compounds (VOCs) in human breath are useful methods in screening, diagnosing and detecting various diseases such as lung cancer, intestinal tract,

\footnotetext{
*Corresponding author.

Email address: dv.an@vju.ac.vn
}

https//doi.org/ 10.25073/2588-1124/vnumap.4457 
asthma, gastric cancer etc. at early stage [1]. A mount of breath VOCs have been identified by various studies as markers of different systemic diseases. 2-butanone (ethyl methyl ketone) is one of the main VOCs which was detected in the breath of patients with Helicobacter pylori (H. Pylori), lung cancer [2] or ovarian cancer [3]. H. Pylori has been associated with colorectal polyps and colorectal cancer. H. Pylori is also linked to the development of duodenal ulcers and stomach cancer. Early detection of $H$. Pylori infection has been an important research topic in contemporary medical institutes for initiation of proper treatment. Several analytical techniques have been developed to measure the concentration of 2-butanone, such as gas chromatography (GC) coupled with mass spectrometry (MS) method, near infrared spectroscopy and sensor. Among these, sensors may be the most practical method since its convenience, cheapness and accuracy.

In recent years, development of variable gas sensing conductors based on the different 2D nanomaterials has attracted great interest in the field of breath diagnostics. These sensing devices are portable, compact and inexpensive with high accuracy for quick results. To be used in the breath analysis, these sensors need the high sensitivity and good selectivity with respect to various different VOCs. The problem can only be solved if the mechanism of gas adsorption is explicitly explored, the suitable gas-sensitive materials are applied and the developing appropriate sensor structures as well as operation of the sensor are optimized. Selecting proper materials for gas sensor is important in increasing the sensitivity, selectivity and stability of the device. 2D materials such as graphene have recently attracted great attention due to their flexible features and high sensitivity to gas adsorption as well as surface phenomena. A portable 2-butanone sensor was experimentally developed by YC. Weng et al. [4] using the graphene or $\mathrm{ZnO}$ electrode. The authors showed that graphene electrode has a shorter response and recovery time upon exposure to 2-butanone when compared with the $\mathrm{ZnO}$ nanorod electrode due to the fast gas transfer into the graphene layers.

In this study, we choose 2-butanone as a maker for diagnosis of $H$. Pylori infection and graphene as a gas sensor material. The adsorption mechanism of 2-butanone on the surface of graphene is investigated by the quantum simulation method. The images of the potential energy surfaces for different positions of the adsorbate on graphene are explored by Computational DFT-based Nanoscope [5] for determination of the most stable configurations and diffusion possibilities. The adsorption energy profiles are calculated by three approximations of van der Waals interactions: revPBE-vdW means the original vdW-DF proposed by Dion et al.[6, 7], the optPBE-vdW functional where the exchange functional was optimized for the correlation part [8], and the vdW-DF2 (a second version of vdW-DF) of Langreth and Lundqvist groups [9]. The vdW-DF and vdW-DF2 functionals using in this study have been implemented by J. Klimes [10] in VASP. These new exchange and correlation functionals were shown to predict better results in the adsorption distances and energies of gas molecules on 2D material surfaces including weak interactions. In addition, current computational investigations can provide deeper insights into the mechanism of 2-butanone adsorption on graphene.

\section{Computational Method}

The adsorption mechanism of 2-butanone on the surface of graphene $4 \times 4$ supercell was investigated by theoretical calculations within the framework of DFT [11, 12]. By using High Performance Computers, $A b$ initio calculation simulation was performed based on the Density Functionals method implemented in the software Vienna Ab initio Simulation Package VASP [13-16]. VASP uses density functionals approaches for solving Schrödinger equation of many-particle systems. This software is a commercial and most popular computational software for the simulation in atomistic scale. 
Graphene built from the geometries obtained from experimental data, is put into a supercell with the single-layered periodicity and with a large vacuum space $(20 \AA)$ to eliminate the possible interaction between layers as shown in Figure 1. The cut-off energy and $K$-mesh [17] in the reciprocal space are optimized for accurate calculations. A cutoff energy of $550 \mathrm{eV}$ for the plane-wave basis set and a Gamma- centered mesh of size $3 \times 3 \times 1$ were utilized. The $K$-point mesh of $8 \times 8 \times 1$ was ensured to yield sufficient energy convergence. All the structures were fully relaxed until the maximum HellmannFeynman force acting on each atom is less than $0.001 \mathrm{eV} / \AA$.

A 2-butanone molecule is initially placed above a carbon atom of graphene, with the $\mathrm{CO}$ functional group oriented parallel or perpendicular to the graphene plane. Orienting possibilities of a 2-butanone molecule on the surface of graphene are systematically investigated using Computational DFT-based Nanoscope tool [5]. Thanks to this tool, the gas molecule is considered as the tip of a scanner by which the molecule moves on the adsorbent surface and rotates around its center of mass to find out the stable adsorption configuration. Using this tool, the problem of handling the complex configurations of VOCs and adsorbents can be solved. The minimum energy configurations will be determined, as well as the optimized distance from VOCs molecules to the adsorbent surface. Using this tool we can search for minimum energy configuration with correction of error if we estimate the adsorption energy by calculating the isolated substrate and adsorbates. The orientation of gas molecules on the surface can be optimized at each configuration. The detailed calculation procedure can be summarized into 2 steps: First, we scan horizontally to determine the stable position of the adsorbate. And second, we scan vertically to determine the adsorption energy profile.

Adsorption energies are calculated, taking into account the contribution from weak interactions such as van de Waals. The calculated results are compared with experimental data to check the reliability of the simulation model. By using Bader charge analysis method [18, 19], the charge transfer between 2butanone and graphene is evaluated and analyzed.

\section{Results and Discussion}

\subsection{Binding Potentials, Stable Position and Adsorption Energy of 2-butanone Molecule on Graphene}

The stable position of 2-butanone can be deduced from the minima on the Potential Energy Surface (PES) as shown in Figure 2. The color gradient represents the relative energy, with dark, black regions having low energy (favorable), and bright, yellow having high energy (unfavorable). The possible diffusion pathways are shown in Figure 2, $x$ and $y$ are fractional coordinates based on the lattice vectors of the xy-plane of the supercell illustrated by Figure 1. The PES in Figure 2a, 2b showed the localized adsorption area for both cases, with the $\mathrm{CO}$ functional group of a 2-butanone molecule oriented parallel and perpendicular to the graphene plane. The dark regions indicate the diffusion paths and the adsorption areas, whereas the bright regions indicate that the 2-butanone molecule is less preferable to get adsorbed on the graphene surface as shown in figures. The estimated diffusion barrier of a system of a 2-butanone molecule oriented parallel to the graphene plane is rather low, which is approximately $4 \mathrm{meV}$. In the case of a 2-butanone molecule oriented perpendicular to the graphene plane, the diffusion barriers are estimated as $45 \mathrm{meV}$ and $4 \mathrm{meV}$.

The difference in diffusion barrier will affect the sensor response. In order to detect the change in the electric current signal, the change in the resistance should be sufficient. The resistance of the sensor gradually changes as more and more VOCs are adsorbed on the surface of graphene. The 2D projected PES is superimposed onto the graphene supercell as shown in Figure 3. Black circles represent carbon graphene atoms and black segments are the bonds connecting them. The energies are shifted so that the 
most stable position corresponds to zero. The $X$ and $Y$ axes are the Cartesian coordinates of XY-plan in units of Angstrom. It can be seen that the 2D projected PES possesses the $4 \times 4$ periodicity due to the periodicity of the graphene supercell. The 3D local PES provides a depth perception of the energy landscape while the 2D projected PES helps to visualize stable positions.

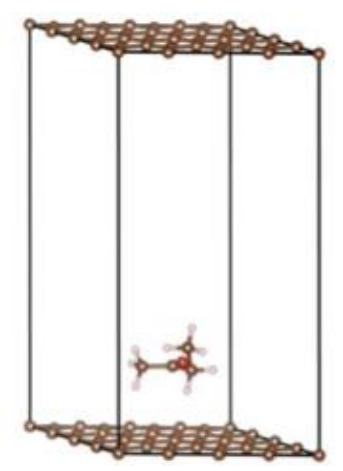

Figure 1. Slab model is built from the graphene $4 \mathrm{x} 4$ supercell. A 2-butanone molecule is initially placed above a carbon atom of graphene, with the $\mathrm{CO}$ functional group oriented parallel to the graphene plane.

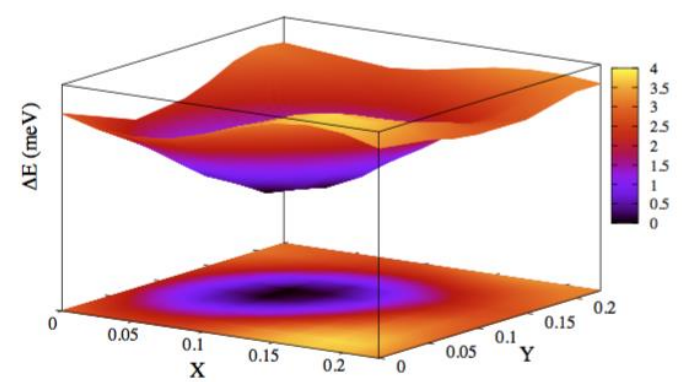

$2 \mathrm{a}$

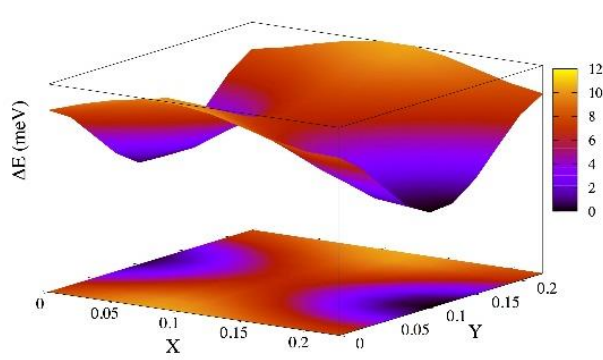

$2 b$

Figure 2. Binding potentials (3D local PES) and diffusion possibility of 2-butanone molecule on graphene,

2a) 2-butanone molecule oriented parallel to the graphene plane; 2b) 2-butanone molecule oriented perpendicular to the graphene plane. The $\mathrm{x}$ and $\mathrm{y}$ axes are fractional coordinates. The origin of $\mathrm{x}$ and $\mathrm{y}$ axes is the initial position of the center of mass of 2-butanone molecule. The $\mathrm{z}$ axis is the energy values which is shifted so that the most stable position corresponds to zero.

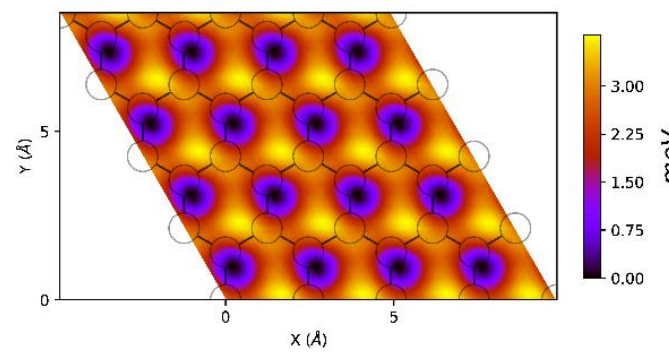

$3 \mathrm{a}$

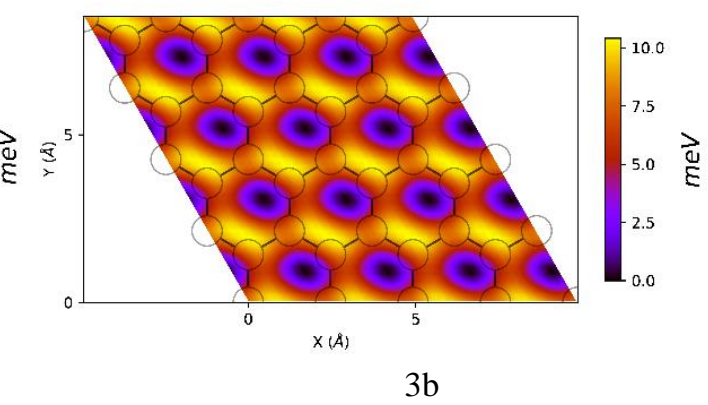

$3 b$

Figure 3. Binding potentials (2D projected PES) of 2-butanone on graphene. 3a) 2-butanone molecule oriented parallel to the graphene plane; 3b) 2-butanone molecule oriented perpendicular to the graphene plane. The X and $\mathrm{Y}$ axes are the Cartesian coordinates of XY-plan in units of Angstrom. 
Next, to investigate further into the interaction between the substrate and the adsorbate, we explore the adsorption energy profile of a 2-butanone molecule on graphene. We use a formulation to calculate the adsorption energy. Adsorption energy and response distance of 2-butanone on graphene vs different Van de Waals interactions are shown in Figure 4 . The $x$-axis represents the distance $(\AA)$ from the center of mass $(\mathrm{COM})$ of the gas molecule to the graphene surface. The $y$-axis corresponds to the adsorption energy, which is defined as:

$$
E_{\text {adsorption }}=E_{\text {complex }}-E_{\text {saturation }}
$$

in which $E_{\text {complex }}$ is the total energy of the system (2-butanone and graphene), $E_{\text {saturation }}$ is the total energy of the configuration of the complex system when a 2-butanone molecule and graphene are isolated from each other. This formula is equivalent to

$$
E_{\text {adsorption }}=E_{\text {complex }}-E_{\text {gas }}-E_{\text {graphene }}
$$

where $E_{\text {gas }}$ and $E_{\text {graphene }}$ are total energies of 2-butanone and graphene, respectively. As we can see in Figure 4, the adsorption energy increases in the order vdW-DF2 < revPBE-vdW < optPBE-vdW.

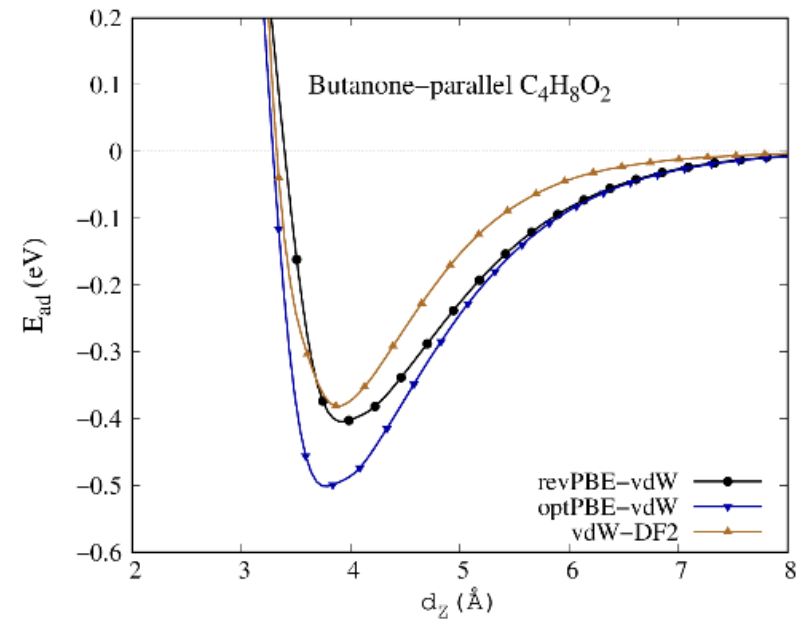

$4 \mathrm{a}$

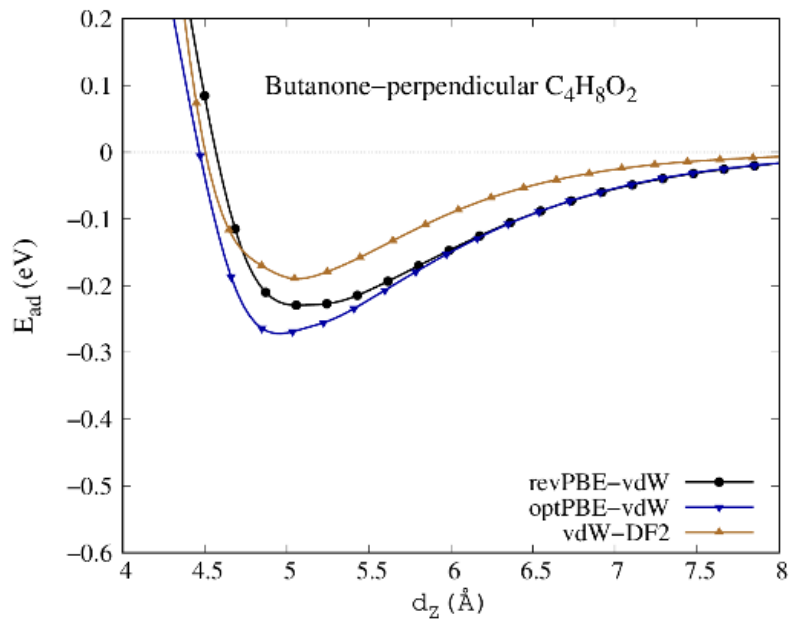

$4 \mathrm{~b}$

Figure 4. Adsorption energy and response distance of 2-butanone molecule oriented parallel and perpendicular to the graphene on graphene vs. different Van de Waals interactions. The x-axis represents the distance from the center of mass (COM) of a 2-butanone molecule to the graphene surface. The y-axis corresponds to the adsorption energy. 4a) 2-butanone molecule oriented parallel to the graphene plane; 4b) 2-butanone molecule oriented perpendicular to the graphene plane.

Table 1. Characteristics of 2-butanone adsorption on graphene, 2-butanone molecule oriented parallel to the graphene plane.

\begin{tabular}{llll}
\hline Dispersion/ Characteristics & revPBE-vdW & optPBE-vdW & vdW-DF2 \\
\hline Distance dz $(\AA)$ & 2.739 & 2.616 & 2.405 \\
Distance cdz $(\AA)$ & 4.023 & 3.858 & 3.590 \\
Response length $(\AA)$ & 8.033 & 8.352 & 7.810 \\
Adsorption Energy $(\mathrm{meV})$ & 400 & 498 & 376 \\
\hline
\end{tabular}


Table 2. Characteristics of 2-butanone adsorption on graphene, 2-butanone molecule oriented perpendicular to the graphene plane.

\begin{tabular}{llll}
\hline Dispersion/Characteristics & revPBE-vdW & optPBE-vdW & vdW-DF2 \\
\hline Distance dz ( $\mathrm{\AA})$ & 2.949 & 2.931 & 2.706 \\
Distance cdz $(\AA)$ & 5.113 & 5.085 & 4.834 \\
Response length $(\AA)$ & 8.149 & 8.131 & 7.414 \\
Adsorption Energy $(\mathrm{meV})$ & 229 & 265 & 189 \\
\hline
\end{tabular}

The characteristics of 2-butanone adsorption on graphene are summarized in Table 1, 2 for a 2butanone molecule oriented parallel to the graphene and a 2-butanone molecule oriented perpendicular to the graphene, respectively. The optimal adsorption distance $d z$ is the distance from the COM of the gas molecule to the graphene surface at full relaxation of the system. This distance is around 4.023$5.113 \AA$ with revPBE-vdW dispersion. We define reponse length as the distance between the gas molecule and the graphene surface that below which the interaction occurs. Response length can be expected to play a role in determining the reponse rate of the sensor. As a 2-butanone molecule descends onto the surface of graphene, a shorter response length might allow faster change in the electronic properties of graphene.

\subsection{Electronic Structure and Charge Transfer Mechanism}

To investigate the nature of 2-butanone adsorption on graphene, we calculated the band dispersion and density of states along high symmetry $k$-points. Based on calculated results shown in Figure 5, the electronic band structures of graphene display gap opening. Band gap energy for the case of a 2butanone molecule oriented parallel to the graphene and a 2-butanone molecule oriented perpendicular to the graphene is $4 \mathrm{meV}$ and $3 \mathrm{meV}$, respectively (Table 3). Opening a band gap might lead to the decreasing of electrical conductivity, which implies the possibility of detecting VOCs by monitoring the conductance of graphene upon exposure to a breath contaning VOCs. The larger the band gap, the larger the change in electrical conductance. Gap opening also implies lower carrier concentration, driving the resistance upward.

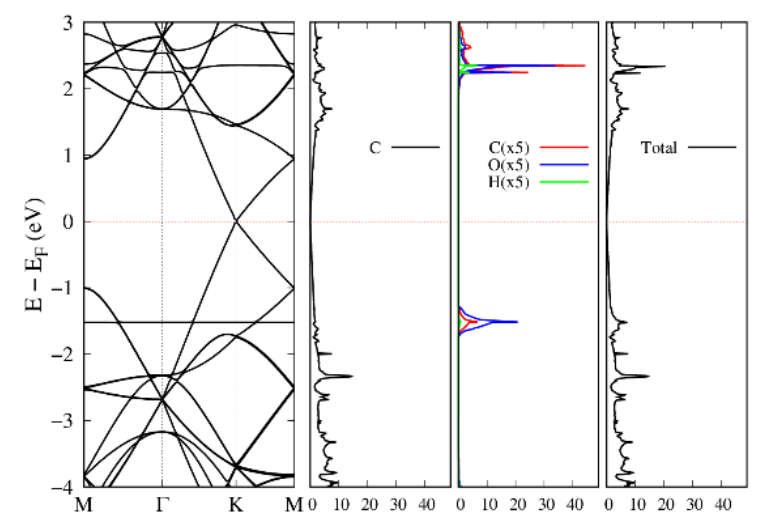

$5 \mathrm{a}$

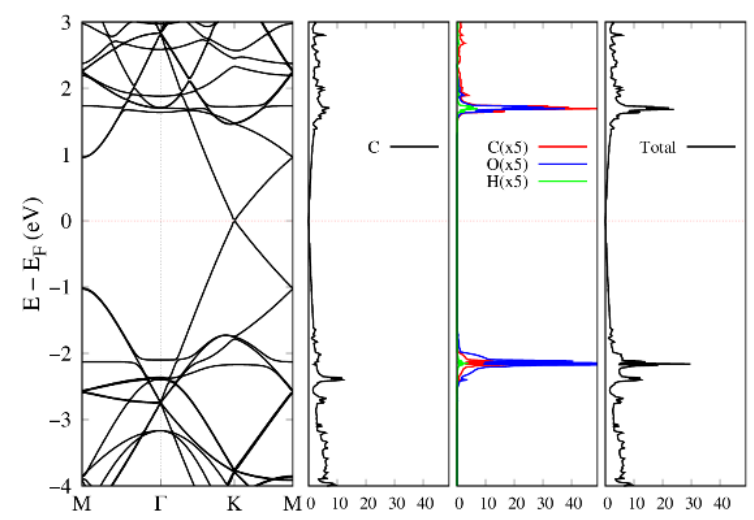

$5 b$

Figure 5. Band structures and density of states (DOS) of 2-butanone on graphene (revPBE-vdW functional). 5a) $\mathrm{CO}$ oriented parallel to the graphene plane; 5b) $\mathrm{CO}$ oriented perpendicular to the graphene plane. The dashed lines represent Fermi level. DOS is in units of state/eV. 
Next, Bader analysis is performed to predict the charge transfer value. The charge density difference provides information about the charge accumulation and charge depletion regions. The charge density difference of a system $\mathrm{AB}$ is $\Delta \rho=\rho_{A B}-\rho_{A}-\rho_{B}$ where $\rho$ is the charge of the system stored in charge density obtained from self consistent calculations. In some cases, in intuitively tells where bonds are made or destroyed. We need to point out that although different methods besides Bader analysis may give rise to different values in determining the electronic charge transfer, the direction and order of magnitude should be the same. By comparison of the charge before and after adsorption of VOC molecules, the magnitude and direction of charge transfer can be inferred. The results for charge transfer analysis of a 2-butanone molecule on graphene are shown in Table 3. The charge density differences induced by the adsorption of 2-butanone on graphene are plotted in Figure 6 and Figure 7.

Table 3. The revPBE-vdW results of the band gap energy and the charge transfers from 2-butanone to graphene.

\begin{tabular}{lll}
\hline \hline 2-butanone/graphene & Parallel & Perpendicular \\
\hline Band gap (meV) & 4 & 3 \\
Charge transfer (e) & 0.078 & 0.025 \\
\hline
\end{tabular}

A 2-butanone molecule acts as a donor on graphene. We notice that there are differences in the numbers of electrons transferring to graphene and in the distance between 2-butanone and graphene. They are correlated because a smaller distance between 2-butanone and graphene leads to a larger orbital overlap and consequently to more orbital mixing (i.e., a larger charge transfer). Comparing to the adsorption of toxic gases $\left(\mathrm{NH}_{3}, \mathrm{NO}, \mathrm{NO}_{2}, \mathrm{CO}, \mathrm{CO}_{2} \ldots\right)$ on the graphene surface [20], the charge transfer from VOCs as 2-butanone to graphene is larger than those from the toxic gases to graphene $(0.012 \sim 0.028 e)$.

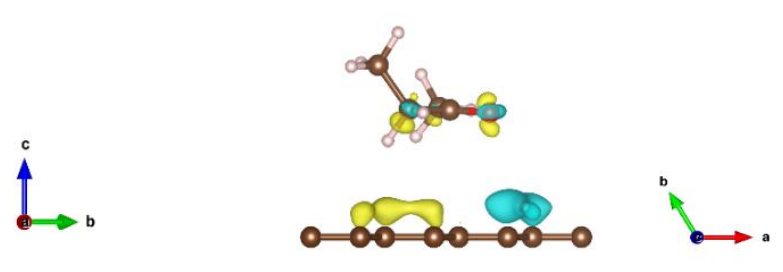

$6 \mathrm{a}$

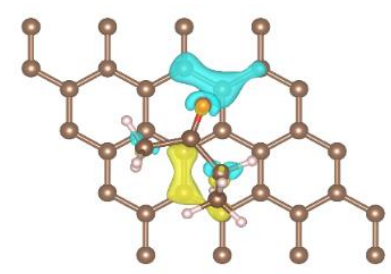

$6 \mathrm{~b}$

Figure 6. a) Side and b) top views of charge density difference induced by the adsorption of 2-butanone on graphene, with the $\mathrm{CO}$ functional group oriented parallel to the graphene plane. The isosurface was set to 0.0004 $\AA^{-2}$, where yellow and blue denote loss and gain of electron density, respectively.

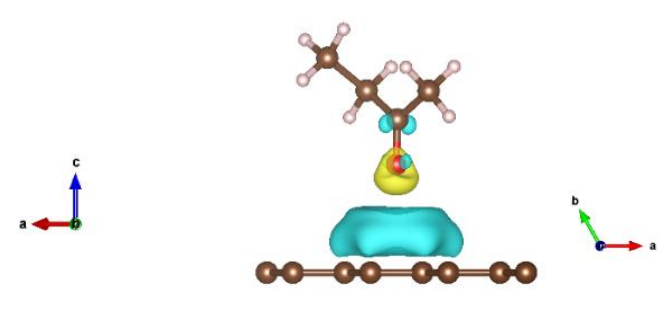

$7 a$

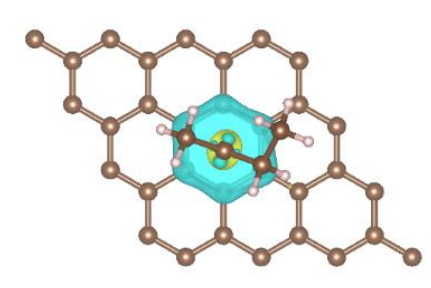

$7 b$

Figure 7. a) Side and b) top views of charge density difference induced by the adsorption of 2-butanone on graphene, with the $\mathrm{CO}$ functional group oriented perpendicular to the graphene plane. The isosurface was set to $0.0003 \AA^{-2}$, where yellow and blue denote loss and gain of electron density, respectively. 
The charge transfer between 2-butanone and graphene is found to be almost independent on the adsorption site but it does depend strongly on the distance of the adsorbate and the graphene surface. There is no linear relationship between the charge transfer magnitude and the adsorption energy or the band gap opening.

\section{Conclusions}

The optimal adsorption position and orientation of a 2-butanone molecule on the graphene surface are determined and the adsorption energies are calculated. The adsorption energy profiles were calculated by three approximations of van der Waals interactions: revPBE-vdW, optPBE-vdW, and vdW-DF2. The adsorption energy ranges from $0.229 \mathrm{eV}$ to $0.4 \mathrm{eV}$. The 2-butanone adsorption on graphene produces a band gap of 3-4 meV. Molecular doping, i.e., charge transfer between a 2-butanone and the graphene surface, is discussed in light of the density of states and the molecular orbitals of the adsorbates. Our calculated results provide a contribution into the database of the adsorption possibility of several categories of some VOCs on pristine graphene and the change of the electronic properties of graphene upon VOC adsorption.

In conclusion, the Potential Energy Surface provides us with the information about the stable positions and possible diffusion channels of a 2-butanone on the surface of graphene. The adsorption energy profile can be used to evaluate some characteristics of VOCs sensor. Finally, from the calculation, we showed that graphene is sensitive to 2-butanone, graphene might have possible application in VOC sensors for detection of cancer through an exhaled breath.

\section{Acknowledgments}

This research was supported by Vietnam National Foundation for Science and Technology Development (NAFOSTED) under Grant Number 103.01-2018.315. The authors gratefully acknowledge the JICA project in Vietnam Japan University for providing the facilities. The research was conducted using the high performance computer system which was invested in part by the investment project on equipment synchronized with facilities construction for education and training at Vietnam National University, Hanoi in Hoa Lac.

\section{References}

[1] Z. Jia, A. Patra, V.K. Kutty, T. Venkatesan, Critical review of volatile organic compound analysis in breath and in vitro cell culture for detection of lung cancer, Metabolites. 9 (2019) 1-17. https://doi.org/10.3390/metabo9030052.

[2] M. Hakim, Y.Y. Broza, O. Barash, N. Peled, M. Phillips, A. Amann, H. Haick, Volatile organic compounds of lung cancer and possible biochemical pathways, Chem. Rev. 112 (2012) 5949-5966. https://doi.org/10.1021/cr300174a.

[3] H. Amal, D. Shi, R. Ionescu, W. Zhang, Q. Hua, Y. Pan, L. Tao, H. Liu, Assessment of ovarian cancer conditions from exhaled breath 136 (2015) E614-E622. https://doi.org/10.1002/ijc.29166.

[4] Y.C. Weng, Y.H. Yang, I.T. Lu, Detection of 2-butanone for the diagnosis of Helicobacter pylori using graphene and $\mathrm{ZnO}$ nanorod electrodes, J. Nanosci. Nanotechnol. 16 (2016) 7077-7084. https://doi.org/10.1166/jnn.2016.10685.

[5] Computational DFT-based Nanoscope tool developed by V. A. Dinh, VNU Vietnam Japan University, 2017.

[6] M. Dion, H. Rydberg, E. Schröder, D.C. Langreth, B.I. Lundqvist, Van der Waals density functional for general geometries, Phys. Rev. Lett. 92 (2004) 22-25. https://doi.org/10.1103/PhysRevLett.92.246401. 
[7] G. Román-Pérez, J.M. Soler, Efficient implementation of a van der waals density functional: Application to doublewall carbon nanotubes, Phys. Rev. Lett. 103 (2009) 1-4. https://doi.org/10.1103/PhysRevLett.103.096102.

[8] J. Klimeš, D.R. Bowler, A. Michaelides, Chemical accuracy for the van der Waals density functional, J. Phys. Condens. Matter. 22 (2010) 22201-22205. https://doi.org/10.1088/0953-8984/22/2/022201.

[9] K. Lee, É.D. Murray, L. Kong, B.I. Lundqvist, D.C. Langreth, Higher-accuracy van der Waals density functional, Phys. Rev. B - Condens. Matter Mater. Phys. 82 (2010) 3-6. https://doi.org/10.1103/PhysRevB.82.081101.

[10] J. Klime, D.R. Bowler, A. Michaelides, Van der Waals density functionals applied to solids, Phys. Rev. B. 83 (2011) 195131(1-13). https://doi.org/10.1103/PhysRevB.83.195131.

[11] W. Kohn, L.J. Sham, Self-Consistent Equations Including Exchange and Correlation Effects, Phys. Rev. 140 (1965) A1133-A1138. https://doi.org/10.1046/j.1365-4362.2002.01376.x.

[12] P. Hohenberg, W. Kohn, Inhomogeneous Electron Gas, Phys. Rev. 136 (1964) B864-B871. https://doi.org/10.1103/PhysRev.136.B864.

[13] David Vanderbilt, Soft self-consistent pseudopotentials in a generalized eigenvalue formalism, Phys. Rev. B. 41 (1990) 7892-7895.

[14] G. Kresse, Ab initio molecular dynamics for liquid metals, J. Non. Cryst. Solids. 192-193 (1995) $222-229$. https://doi.org/10.1016/0022-3093(95)00355-X.

[15] G. Kresse, J. Hafner, Ab initio molecular-dynamics simulation of the liquid-metalamorphous- semiconductor transition in germanium, Phys. Rev. B. 49 (1994) 14251-14269. https://doi.org/10.1103/PhysRevB.49.14251.

[16] G. Kresse, J. Furthmüller, Efficiency of ab-initio total energy calculations for metals and semiconductors using a plane-wave basis set, Comput. Mater. Sci. 6 (1996) 15-50. https://doi.org/10.1016/0927-0256(96)00008-0.

[17] J.D. Pack, H.J. Monkhorst, Special points for Brillouin-zone integrations - a reply, Phys. Rev. B. 16 (1977) 17481749. https://doi.org/10.1103/PhysRevB.16.1748.

[18] G. Henkelman, A. Arnaldsson, H. Jónsson, A fast and robust algorithm for Bader decomposition of charge density, Comput. Mater. Sci. 36 (2006) 354-360. https://doi.org/10.1016/j.commatsci.2005.04.010.

[19] G. Henkelman, B.P. Uberuaga, H. Jónsson, Climbing image nudged elastic band method for finding saddle points and minimum energy paths, J. Chem. Phys. 113 (2000) 9901-9904. https://doi.org/10.1063/1.1329672.

[20] O. Leenaerts, B. Partoens, F.M. Peeters, Adsorption of $\mathrm{H}_{2} \mathrm{O}, \mathrm{NH}_{3}, \mathrm{CO}, \mathrm{NO}_{2}$, and $\mathrm{NO}$ on graphene: A first-principles study, Phys. Rev. B. 77 (2008) 1254161-1254166. https://doi.org/10.1103/PhysRevB.77.125416. 